\title{
LOWER ESTIMATES FOR ZEROS OF STOCHASTIC STURM-LIOUVILLE PROBLEMS
}

\author{
KURT KREITH ${ }^{1}$
}

\begin{abstract}
Letting $T(\omega)$ denote the first zero of a class of stochastic SturmLiouville initial value problems, a lower bound is established for the expected value of the random variable $T$.
\end{abstract}

1. Introduction. For stochastic Sturm-Liouville eigenvalue problems of the form

$$
-\left(p(t, \omega) u^{\prime}\right)^{\prime}+q(t, \omega) u=\lambda u ; \quad u(a)=u(b)=0,
$$

it would be of great interest to be able to determine the distribution of the random variables $\lambda_{n}(\omega)$ from full knowledge of the coefficients. Here $p(t, \omega)$ and $q(t, \omega)$ are sufficiently regular $[\mathbf{1}]$ functions defined on $[a, b] \times \Omega$, where $\Omega$ is the sample space for some fixed probability measure and $p(t, \omega)$ is assumed positive. Closely related is the problem of determining the distribution of the first zero $T(\omega)>a$ of the nontrivial solutions of the stochastic initial value problem

$$
-\left(p(t, \omega) u^{\prime}\right)^{\prime}+q(t, \omega) u=0 ; \quad u(a)=0, u^{\prime}(a)=1
$$

whose coefficients are sufficiently regular and satisfy $p(t, \omega)>0$ on $[a, \infty) \times \Omega$.

Unfortunately, neither of these problems is tractable except in some very special cases-e.g. cases in which solutions of the form $u(t, \omega)$ can be found explicitly. More typically, one must be content with incomplete information about the random variables $\lambda(\omega)$ and $T(\omega)$.

Boyce $[1,2]$ has obtained an interesting upper estimate for the first eigenvalue $\lambda_{1}(\omega)$ of $(1.1)$ in terms of a related deterministic Sturm-Liouville equation. Denoting the expected value of $X(\omega)$ by $\langle X\rangle$ and making appropriate regularity assumptions on $p(t, \omega)$ and $q(t, \omega)$, Boyce $[\mathbf{1}]$ establishes the inequality

$$
\left\langle\lambda_{1}\right\rangle \leq \mu_{1}
$$

where $\mu_{1}$ is the first eigenvalue of the deterministic eigenvalue problem

$$
-\left(\langle p\rangle(t) v^{\prime}\right)^{\prime}+\langle q\rangle(t) v=\mu v ; \quad v(a)=v(b)=0 .
$$

However, for the second eigenvalue the inequality $\left\langle\lambda_{2}\right\rangle \leq \mu_{2}$ need not hold [2].

From classical variational principles for eigenvalues $[\mathbf{3}]$ it is well known that $\lambda_{1}(\omega)$ varies inversely with $T(\omega)$. This fact, combined with Boyce's theorem, suggests the possibility of establishing a corresponding inequality of the form

$$
\langle T\rangle \geq S,
$$

Received by the editors September 19, 1983. Presented at the International Conference on Qualitative Theory of Differential Equations held at the University of Alberta, June 18-20, 1984. 1980 Mathematics Subject Classification. Primary 34F05; Secondary 60H10.

Key words and phrases. Stochastic Sturm-Liouville problem, Jensen's inequality, first zero, first eigenvalue.

${ }^{1}$ Supported by a grant of the National Science Foundation DMS 8318136.

(C) 1984 American Mathematical Society $0002-9939 / 84 \$ 1.00+\$ .25$ per page 
where $S$ is the first zero of the deterministic initial value problem

$$
-\left(\langle p\rangle(t) v^{\prime}\right)^{\prime}+\langle q\rangle(t) v=0 ; \quad v(a)=0, v^{\prime}(a)=1 .
$$

Unfortunately the relationship between $\langle T\rangle$ and $S$ is more complicated than that between $\left\langle\lambda_{1}\right\rangle$ and $\mu_{1}$. In particular, one can readily construct examples (see $\S 2$ ) of equations of the simple form

$$
-u^{\prime \prime}+[q(t)-\rho(\omega)] u=0
$$

for which (1.4) is false.

However, consideration of the example

$$
u^{\prime \prime}+\rho(\omega) u=0 ; \quad u(0)=0, u^{\prime}(0) \neq 0
$$

with solution $u(t, \omega)=A \sin \sqrt{\rho(\omega)} t$ suggests that (1.4) should nonetheless hold for a large class of equations (1.2). In the case of (1.6) we have $T=\pi / \sqrt{\rho}$ so that by Jensen's inequality $\langle T\rangle \geq \pi / \sqrt{\langle\rho\rangle}=S$. The present note shows that this behavior is not atypical by establishing that (1.4), while not as universal as (1.3), does hold in many specified situations.

2. A counterexample. We begin with an example, showing how to construct stochastic Sturm-Liouville equations of the form

$$
u^{\prime \prime}+[\rho(\omega)-q(t)] u=0 ; \quad u(0)=0, u^{\prime}(0) \neq 0
$$

for which (1.4) fails-i.e. for which

$$
\langle T\rangle<S
$$

Let $\rho(\omega)$ satisfy $\operatorname{Pr}\left(\rho=\frac{1}{2}\right)=\operatorname{Pr}\left(\rho=\frac{3}{2}\right)=\frac{1}{2}$ so that $\langle\rho\rangle=1$. We also define

$$
\begin{aligned}
q(t) & =0 & & \text { for } 0 \leq t<\pi / 2 \\
& =1 & & \text { for } \pi / 2 \leq t<M \\
& =0 & & \text { for } M \leq t<\infty
\end{aligned}
$$

In this case $S(M)$ is determined by the following solution of $u^{\prime \prime}+[1-q(t)] u=0$ :

$$
\begin{aligned}
u(x, t) & =\sin t & & \text { for } 0 \leq t \leq \pi / 2 \\
& =1 & & \text { for } \pi / 2 \leq t \leq M \\
& =\sin (t-M+\pi / 2) & & \text { for } M \leq t<\infty
\end{aligned}
$$

so that $S=M+\pi / 2$. To calculate $\langle T\rangle$ we consider separately two cases:

1. If $\rho=\frac{1}{2}$, the solution of (2.1) satisfies $u^{\prime \prime}+\frac{1}{2} u=0$ for $t>M$ so that $T<M+\pi \sqrt{2}$.

2. If $\rho=\frac{3}{2}$ then $\rho-q(t) \geq \frac{1}{2}$ for $t \geq 0$ so that $T<\pi \sqrt{2}$.

Therefore $\langle T\rangle<\frac{M}{2}+\pi / 2$ and (2.2) holds when $M$ is sufficiently large (e.g. for $M \geq 10)$.

While the preceding example utilizes a discontinuous coefficient $q(t)$ and a discrete random variable $\rho(\omega)$, it is clear that both $q(t)$ and the distribution function for $\rho(t)$ can be mollified without destroying the inequality (2.2). 
3. Lower bounds for $\langle T\rangle$. In light of the example posed by (1.6), a rather natural approach to estimating the first positive zero of

$$
-\left(p(t) u^{\prime}\right)^{\prime}+[q(t)-\rho(\omega)] u=0 ; \quad u(0)=0, u^{\prime}(0)=1
$$

is to denote the first eigenvalue of

$$
-\left(p(t) u^{\prime}\right)^{\prime}+q(t) u=\sigma u ; \quad u(0)=u(\tau)=0 ; \quad \tau>0
$$

by $\sigma_{1}(\tau)$ and to observe that $\rho(\omega)=\sigma_{1}(T)$. Since classical variational principles imply that $d \sigma_{1} / d \tau<0$ for all $\tau>0$, we can solve this equation for $T=h(\rho)$. If $h$ is a convex function, then by Jensen's inequality we again have $\langle T\rangle \geq h(\langle\rho\rangle)=S$.

While this approach may provide a useful computational technique, the complexity of the relationship betwen $T$ and $\rho$ makes it of limited theoretical value. Unless the solution of (3.2) can be found explicitly, one is forced to determine $h(\rho)$ by means of a technique such as the Prüfer transformation $\theta=\tan ^{-1}\left(u / p u^{\prime}\right)$. This substitution in (3.2) leads to

$$
\theta_{t}(t, \sigma)=\frac{1}{p} \cos ^{2} \theta+(\sigma-q) \sin ^{2} \theta ; \quad \theta(0, \sigma)=0
$$

whose solution then defines $\sigma_{1}(T)$ implicitly by means of the equation $\theta(T, \sigma)=$ $\pi$. While the Prüfer transformation can provide useful insight into asymptotic properties of solutions of (3.2), it does not seem to provide an effective theoretical means of establishing the convexity of $h(\rho)$.

These observations, together with the fact that the convexity of $h(\rho)$ is a sufficient rather than necessary condition for $\langle T\rangle \geq S$, suggest the consideration of other techniques. In particular, we consider the case (note the sign change!) of:

$$
\left(p(t, \omega) u^{\prime}\right)^{\prime}+q(t, \omega) u=0 ; \quad u(0)=0, u^{\prime}(0)=1
$$

and

$$
\left(\langle p\rangle(t) u^{\prime}\right)^{\prime}+\langle q\rangle(t)=0 ; \quad u(0)=0, u^{\prime}(0)=1
$$

where the coefficients of (3.4) satisfy inequalities of the form

$$
0<p_{2}(\omega) \leq p(t, \omega) \leq p_{1}(\omega), \quad 0<q_{1}(\omega) \leq q(t, \omega) \leq q_{2}(\omega),
$$

for all $(t, \omega) \in[0, \infty) \times \Omega$. In this situation we have the following result.

THEOREM. Suppose the coefficients of (3.4) satisfy (3.6) and let $T(\omega)$ denote the first positive zero of (3.4). If

$$
\left\langle\sqrt{p_{2} / q_{2}}\right\rangle \geq \sqrt{\left\langle p_{1}\right\rangle /\left\langle q_{1}\right\rangle}
$$

then $\langle T\rangle \geq S$, where $S$ is the first positive zero of (3.5).

ProOF. By (3.6) the differential equations

$$
p_{1}(\omega) u_{1}^{\prime \prime}+q_{1}(\omega) u_{1}=0 ; \quad u_{1}(0)=0, u_{1}^{\prime}(0)=1
$$

and

$$
p_{2}(\omega) u_{2}^{\prime \prime}+q_{2}(\omega) u_{2}=0 ; \quad u_{2}(0)=0, u_{2}^{\prime}(0)=1
$$

are, respectively, Sturmian minorants and majorants for (3.4) [4]. If $T_{1}(\omega)$ and $T_{2}(\omega)$ denote their first zeros, then

$$
T_{1}(\omega) \geq T(\omega) \geq T_{2}(\omega)
$$


for all $\omega \in \Omega$. Since

$$
u_{i}(t)=A_{i} \sin \sqrt{q_{i} / p_{i}} t ; \quad i=1,2,
$$

we have $T_{i}=\pi \sqrt{p_{i} / q_{i}}$ and $S_{i}=\pi \sqrt{\left\langle p_{i}\right\rangle /\left\langle q_{i}\right\rangle}$, so that our hypothesis (3.7) becomes $\left\langle T_{2}\right\rangle \geq S_{1}$. In light of (3.8) we therefore conclude that $\langle T\rangle \geq\left\langle T_{2}\right\rangle \geq S_{1} \geq S$ as was to be shown.

REMARK. The sign condition $q_{1}(\omega)>0$ in (3.6) is only a convenience to assure that all quantities in (3.7) are defined. If (3.7) remains valid without this restriction, we may allow sign-indefinite $q_{i}(\omega)$ and $q(t, \omega)$ in the above theorem.

4. Translated uniform distributions. In applying the preceding theorem it is important to have an estimate for the latitude which (3.6) allows in the coefficients of (3.4). By way of example, we restrict our attention to the case $p(t, \omega) \equiv 1$ and seek to satisfy $(3.6)$ in terms of a constant random variable $\rho(\omega)$ for which $0<\rho(\omega)-c \equiv q_{1}(\omega) \leq q(\omega, t) \leq q_{2}(\omega) \equiv \rho(\omega)$. The problem at hand is then to determine the largest value of $c$ for which (3.7) remains valid-i.e., for which

$$
\left\langle\frac{1}{\sqrt{\rho}}\right\rangle \geq \sqrt{\frac{1}{\langle\rho\rangle-c}}
$$

On the basis of such a calculation one could conclude, for example, that the first zero of

$$
u^{\prime \prime}+\left[q_{0}(t)+\rho(\omega)\right] u=0 ; \quad u(0)=0, u^{\prime}(0)=1
$$

satisfies $\langle T\rangle \geq S$ whenever $k-c \leq q_{0}(t) \leq k$ for some constant $k$.

The constant $c$ can be computed explicitly in the case where $\rho$ is uniformly distributed on $[a, b]$. Here

$$
\left\langle\frac{1}{\sqrt{p}}\right\rangle=\frac{1}{b-a} \int_{a}^{b} x^{-1 / 2} d x=\frac{2}{\sqrt{a}+\sqrt{b}}
$$

while

$$
\sqrt{\frac{1}{\langle\rho\rangle-c}}=\sqrt{\frac{2}{a+b-2 c}}
$$

from which it follows readily that (4.1) is satisfied whenever $c \leq \frac{1}{4}(\sqrt{a}-\sqrt{b})^{2}$.

This example suggests that increasing the degree of randomness in the coefficients also enlarges the class of differential equations for which (1.4) is valid.

\section{REFERENCES}

1. W. E. Boyce, Random eigenvalue problems, Probabilistic Methods in Applied Mathematics (A. T. Bharucha-Reid, ed.), Academic Press, New York, 1968.

2. _ On a conjecture concerning the means of the eigenvalues of random Sturm-Liouville boundary value problems, Quart. Appl. Math. 38 (1980), 241-245.

3. R. Courant and D. Hilbert, Methods of mathematical physics, vol. 1, Wiley, New York, 1953.

4. C. A. Swanson, Comparison and oscillation theory of linear differential equations, Academic Press, New York, 1968.

Department of Mathematics, University of California, Davis, California 